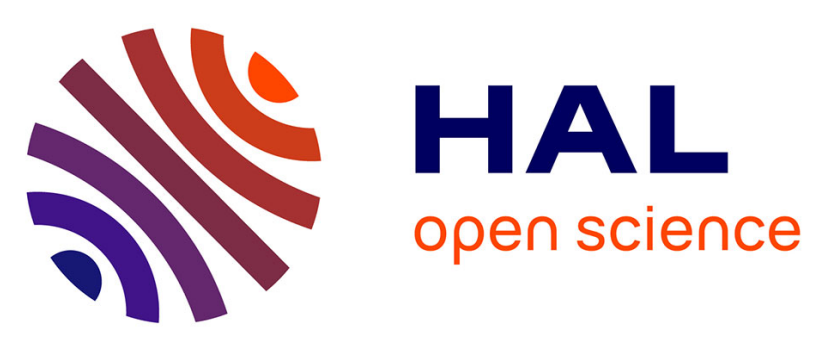

\title{
Multiuser Time Reversal UWB Communication System: A Modified Transmission Approach
}

\author{
Ijaz Haider Naqvi, Ali Khaleghi, Ghaïs El Zein
}

\section{To cite this version:}

Ijaz Haider Naqvi, Ali Khaleghi, Ghaïs El Zein. Multiuser Time Reversal UWB Communication System: A Modified Transmission Approach. The 20th IEEE International Symposium on Personal, Indoor and Mobile Radio Communications, (PIMRC 2009), Sep 2009, Tokyo, Japan. pp.1-5. hal00448870

\section{HAL Id: hal-00448870 \\ https://hal.science/hal-00448870}

Submitted on 20 Jan 2010

HAL is a multi-disciplinary open access archive for the deposit and dissemination of scientific research documents, whether they are published or not. The documents may come from teaching and research institutions in France or abroad, or from public or private research centers.
L'archive ouverte pluridisciplinaire HAL, est destinée au dépôt et à la diffusion de documents scientifiques de niveau recherche, publiés ou non, émanant des établissements d'enseignement et de recherche français ou étrangers, des laboratoires publics ou privés. 


\title{
Multiuser Time Reversal UWB Communication System: A Modified Transmission Approach
}

\author{
I. H. Naqvi, A. Khaleghi, G. El Zein \\ Institute of Electronics and Telecommunications of Rennes, IETR-UMR CNRS 6164 \\ INSA, 20 Avenue des Buttes de Coësmes, 35043, Rennes, France. \\ ijaz-haider.naqvi@insa-rennes.fr, ali.khaleghi@rr-research.no,ghais.el-zein@insa-rennes.fr
}

\begin{abstract}
In this paper, ultra-wideband (UWB), time reversal (TR) communication is investigated by modifying the transmission prefilter. Mathematical expressions for received signal and the interference in the modified transmission scheme are derived. It is shown that the modified transmission approach reduces multi-user interference which eventually translates into a better bit error rate (BER) performance than simple TR multiuser scheme. Channel impulse responses (CIR) of a typical indoor channel are measured. In a multi-user scenario, both TR and the modified TR schemes are studied using the measured CIRs. It is shown that the proposed modified TR scheme outperforms the original TR scheme.

Index Terms-Multiuser, time reversal (TR), dense multipath propagation channel, ultra-wideband (UWB), bit error rate (BER).
\end{abstract}

\section{INTRODUCTION}

Pulsed Ultra-Wideband (UWB) communication system can achieve high data rate due to its ultra large bandwidth. The ability of pulsed UWB to resolve individual multi-path components is exploited in the recent research for short range communication applications. However, large number of resolvable paths and low power limitations necessitate a complex receiver system. To collect the received signal energy, Rake receiver, transmit-reference or decision feedback autocorrelation receiver can be implied [1]-[3]. Each technique has different difficulties and drawbacks. The characteristics of an Impulse radio and use of Rake receivers for signal detection has already been studied for communication in dense multipath environment [4],[5].

Time Reversal (TR) has been proposed as a technique to shift the design complexity from the receiver to the transmitter. Classically, TR has been applied in acoustics and under water communication applications [6],[7], but recently, it has been widely studied for UWB communication [8]-[16]. The received signal in a TR system is considerably focused in spatial and temporal domains. As a result, the received power is concentrated within few taps and the effects of inter symbol interference (ISI) are greatly reduced. The receiver system becomes simpler than without TR and signal can be collected using a simple energy threshold detector [8]. Spatial focusing enables low multi-user and co-channel interference in a multiuser and multi cell systems respectively.

${ }^{1}$ Work supported by ANR project MIRTEC and French research ministry.
In [8], TR filter was modified using circular shift operation in a multiuser scenario. Inherent limitations of the scheme were identified in [17]. In this paper, a further modified transmission scheme for the TR system is proposed for a multiuser TR communication system. The proposed scheme addresses these limitations and gives a significantly better performance than the other schemes. The improvement in the performance increases with the increase in the number of users. Mathematical expressions for received signal and the interference of the proposed transmission scheme are derived. Experiments are performed in a typical indoor environment and results show that the proposed scheme outperforms the original TR scheme. Maximum number of simultaneous users increases with the proposed scheme. Expression for maximum number of simultaneous users is proposed.

The rest of the paper is organized as follows. A brief introduction of the TR scheme is presented in the Section II. The description of the proposed transmission scheme is presented in Section III. Experimental measurement setup, simulation results for the performance of the proposed transmission scheme are presented and analysed in Section IV. Finally, Section V concludes this paper.

\section{TIME REVERSAL}

Time reversal (TR) is a transmission scheme in which a time reversed channel impulse response (CIR) is used as a transmitter pre-filter. In a first step the CIR is estimated between an emitter and a receiver. The CIR is then flipped in time and transmitted in the same channel. The time reversed wave back propagates in the channel following the same paths as the CIR's ones but in the reverse order. Finally at the receiver, all the paths add up coherently in the time and spatial domains. For dense multipath propagation channels, strong temporal compression and high spatial focusing can be achieved with a focusing gain of about $8 \mathrm{~dB}$ [15]. For communication purposes, this gain improves, for instance, the transmission range. Inter Symbol Interference (ISI) effects are mitigated by temporal compression and multiuser interference is reduced due to spatial focusing. The received signal $\left(y_{j}(t)\right)$ at the intended receiver $(j)$ can be mathematically represented as:

$$
y_{j}(t)=s(t) \star h_{i j}(-t) \star h_{i j}(t)=s(t) \star R_{i j}^{a u t o}(t)
$$


where $h_{i j}(t)$ is the CIR from the transmitting point $(i)$ to an intended receiver $(j), s(t)$ is the transmitted signal, $\star$ denotes convolution product and $R_{i j}^{a u t o}(t)$ is the autocorrelation of the CIR, $h_{i j}(t)$. The received signal at any non intended receiver $(k)$ is written as:

$$
y_{k}(t)=s(t) \star h_{i j}(-t) \star h_{i k}(t)=s(t) \star R_{i k j}^{c r o s s}(t)
$$

where $h_{i k}(t)$ is the CIR from the transmitting point $(i)$ to an unintended receiver $(k)$ and $R_{i k j}^{\text {cross }}(t)$ is the cross-correlation of the CIR $h_{i k}(t)$ and $h_{i j}(t)$. If the channels are uncorrelated, then the signal transmitted for one receiver will act as a noise for a receiver at any other location. Thus, a secure communication is achieved with low probability of detection and low probability of interception.

In the practical implementation of the TR system, the precoding filter is truncated in time to reduce the filter length and thus the system complexity. The truncated response is represented as $h^{\prime}(-t)$. For data communication purpose, the transmitted symbols are modulated by binary pulse amplitude modulation (BPAM) scheme. The $k^{t h}$ symbol, $d_{k}$, of the symbol sequence is equal to 1 or -1 for the data bits 1 or 0 respectively. Therefore, the received signal at the intended receiver is written as:

$$
\begin{array}{r}
y(t)=\underbrace{A \sum_{k} d_{k} h^{\prime}\left(-t-k T_{s}\right)}_{\text {Transmitted } \mathrm{RF} \text { signal }} \star \underbrace{h_{(}(t)}_{\mathrm{CIR}}+n(t) \\
\approx A \sum_{k} d_{k} R_{h h}\left(t-k T_{s}\right)+n(t)
\end{array}
$$

where $\mathrm{A}$ is a normalization factor and $T_{s}$ is the inter-symbol interval. $n(t)$ is the noise and $R_{h h}(t)$ is the autocorrelation function of the CIR $(h(t))$. For the sake of simplicity, we have supposed that the $T_{s}=T_{\text {sig }}$, where $T_{\text {sig }}$ is the length of the measured time reversed CIR $\left(h^{\prime}(-t)\right)$ in seconds. As the amplitude of the peak of the received signal is proportional to the energy of the transmitted signal $\left(\int h^{\prime 2} d t\right)$, the truncation process decreases the peak of the received signal. Due to BPAM, the polarity of the received signal peaks depends on the transmitted data bit and is used for the detection of the data bits.

\section{DESCRIPTION OF THE PROPOSED TRANSMISSION APPROACH}

In a multi user TR system, multiple signals for different users are transmitted simultaneously. Let the measured and truncated CIR from the transmitter to the user $i$ can be written as:

$$
h_{i}^{\prime}(-t)=\sum_{m=0}^{L-1} a_{m} \delta\left(t-m \tau_{s}\right)
$$

where $L$ is number of time reversal filter taps which corresponds to $T_{s i g}=L \tau_{s}$ in time(seconds), $a_{m}$ is the associated amplitude and $m \tau_{s}$ is the associated delay of the multi-path components. $\tau_{s}$ is the time between two consecutive sample and depends on the sampling rate of the time reversal filter.
For instance, if the measured CIR is sampled with a sampling rate of $5 \mathrm{GS} / \mathrm{s}$, the delay of $\tau_{s}=0.2 \mathrm{~ns}$ is obtained between two consecutive samples, therefore the number of taps (L) in filter having a length of $50 \mathrm{~ns}$ is $L=250$ samples or taps. The transmitted signal for multi user TR can be written as:

$$
T_{x}(t)=\sum_{k} \sum_{i=0}^{N_{u}-1} \frac{1}{\sqrt{N_{u}}} d_{i k} A_{i} h_{i}^{\prime}\left(-t-k T_{s}\right)
$$

where $d_{i k}$ is the $k_{t h}$ information bit of the $i_{t h}$ user, $N_{u}$ is the total number of simultaneous user, $T_{s}$ is the inter symbol interval and $A_{i}$ is the normalization factor which can be written as:

$$
A_{i}=\frac{1}{\sqrt{\left\|h_{i}^{\prime}\left(-t-k T_{s}\right)\right\|^{2}}}
$$

where $\|\cdot\|$ is the Frobenius norm operation. The term $\frac{1}{N_{u}}$ insures that the signals for all the users are transmitted with a constant power. This is in contrast to [8], where this normalization has not been carried out resulting in an unfair comparison of the performance with different number of users. The received signal for the $j_{t h}$ user and the $k_{t h}$ symbol can be written as:

$$
\begin{gathered}
R_{x_{k j}}(t)=\underbrace{\frac{1}{\sqrt{N_{u}}} d_{j k} h_{j}(t) \star h_{j}^{\prime}\left(-t+j T_{u}-k T_{s}\right)}_{\text {Signal }} \\
+\underbrace{\frac{1}{\sqrt{N_{u}}} \sum_{i=0, i \neq j}^{N_{u}-1} d_{i k} h_{j}(t) \star h_{i}^{\prime}\left(-t+i T_{u}-k T_{s}\right)}_{\text {Interference }}
\end{gathered}
$$

The responses of the modified filter are produced by shifting $h^{\prime}(-t)$ to either left or right and then forcing the shifted part to zero so that the shifted signal can be packed in the same signal duration. Fig. 1 shows the pattern of the left or right shift for $l=1,2$ taps. As shown for the left shift of 1 tap, the last three taps are shifted to left by one tap and the slot for last tap is filled with zero. For the right shift of 1 tap, first three taps are shifted to right and then the slot for the first tap is filled with zero. With the process of filling the shifted taps with zeros, we get rid of the unwanted signal, which causes interference to the adjacent symbols (called Image in [17]). As the taps which result in an undesired signal are forced to zero, the received peak signal increases for an equal transmitted power. If $h^{\prime}(-t)$ is shifted left by $T_{u}=l \tau_{s}$, the expression is given by:

$$
\begin{array}{r}
\text { left shift }\left(h^{\prime}(-t), T_{u}\right)=\hat{h}\left(-t+T_{u}\right) \\
=\left[\sum_{i=0}^{L-l-1} a_{i+l} \delta\left(t-i \tau_{s}\right) z \operatorname{eros}(1, l)\right]
\end{array}
$$

where $z \operatorname{eros}(1, l)$ is a vector containing $l$ number of zeros, $l$ is the number of taps required to carry out a shift of $T_{u}$ seconds. Note that $\hat{h}\left(-t+T_{u}\right)$ has $L-l$ non zero taps. The 

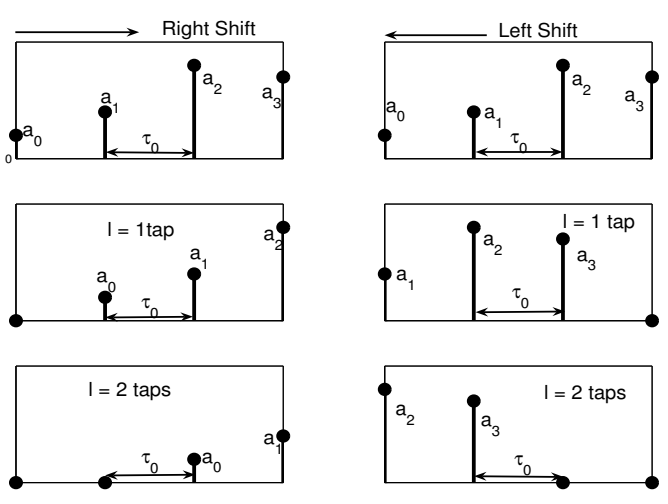

Fig. 1. Pattern for the left and right shift

transmitted signal with the modified transmission scheme can be thus written as:

$$
T_{x}(t)=\sum_{k} \sum_{i=0}^{N_{u}-1} \frac{1}{\sqrt{N_{u}}} d_{i k} A_{i} \hat{h_{i}}\left(-t+i T_{u}-k T_{s}\right)
$$

where $A_{i}=\frac{1}{\sqrt{\left\|\hat{h}\left(-t+i T_{u}-k T_{s}\right)\right\|^{2}}}$ and the term $\frac{1}{\sqrt{N_{u}}}$ insures that power transmitted for different number of users is constant. The received signal for the $k_{t h}$ symbol of the $j_{t h}$ user can be written as:

$$
\begin{gathered}
R_{x_{k j}}(t)=\underbrace{\frac{1}{\sqrt{N_{u}}} d_{j k} h_{j}(t) \star \hat{h_{j}}\left(-t+j T_{u}-k T_{s}\right)}_{\text {Signal }} \\
+\underbrace{\frac{1}{\sqrt{N_{u}}} \sum_{i=0, i \neq j}^{N_{u}-1} d_{i k} h_{j}(t) \star \hat{h}_{i}\left(-t+i T_{u}-k T_{s}\right)}_{\text {Interference }}
\end{gathered}
$$

\section{A. Interference Analysis of the Proposed Scheme}

To analyze the worst case performance, it is assumed that the transmitter communicates with all users at the same time. Therefore, TR received signal in a multi user scenario consists of a sum of one autocorrelation function and $N_{u}-1$ cross correlation functions. The $N_{u}-1$ cross correlation functions cause multi user interference. The received signal peak is the sum of the square of the coefficients of CIR (from the properties of auto correlation function). The interference term at the time of the peak $\left(t_{\text {peak }}\right)$ for the $j_{t h}$ user in a simple TR scheme can be written as:

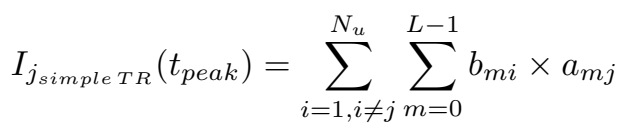

where $b_{m i}$ are the coefficients of the time reversed CIR $h_{i}^{\prime}\left(-t+i T_{u}-k T_{s}\right)$ (from 7)) specific to the user $i, a_{m j}$ are the coefficients of $h_{j}(t)$. As (11) uses simple TR transmission scheme, the high powered taps of $h_{i}^{\prime}(-t)$ and $h_{j}^{\prime}(-t)$ are approximately in the same time interval. Therefore, taps in the propagating channel containing more energy are multiplied by the taps of the transmitted signal with more energy resulting in a relatively higher interference.

To calculate the interference of the modified transmission scheme, we assume that the transmitted signal for the intended receiver is not shifted. Then by using (10) and (11), interference at the peak of the intended signal is then written as:

$$
I_{j_{\text {mod.TR }}}\left(t_{\text {peak }}\right)=\sum_{i=0, i \neq j}^{N_{u}-1} \sum_{m=0}^{L-l-1} b_{(m+l) i} \times a_{m j}
$$

where $b_{m i}$ and $a_{m j}$ are the same coefficients used in (11). Here the interference is the product of $L-l$ coefficients (as $l$ taps are forced to zero). Furthermore, (12) suggests that in case of modified TR scheme, the taps in the propagating channel containing more energy are multiplied by the taps of the transmitted signal with less energy and vice versa. Therefore, interference is reduced with the new proposed transmission scheme. This reduced interference helps to improve the bit error rate (BER) performance of the system.

\section{B. Effects of Shift on Received Signal Peak}

In a TR communication system, as the received signal is the auto correlation function of the CIR, the received signal peak is the sum of the square of the coefficients of CIR. Neglecting the interference and the noise, the received signal peak with the modified TR scheme for the $k_{t h}$ symbol of the $j_{t h}$ user is written as:

$$
R_{x_{\text {modTR }}}\left(t_{\text {peak }_{j k}}\right)=d_{j k} A_{j}\left(\sum_{m=0}^{L-l_{j}-1} a_{m}^{2}\right)
$$

where $a_{i}$ are the coefficients of taps of $\hat{h}\left(-t+j T_{b}-k T_{s}\right)$ and $l_{j}\left(j \frac{T_{b}}{\tau_{s}}\right)$ are the number of taps required for a shift of $j T_{b}$. The received signal peak depends on the energy contents of $\left(L-l_{j}\right)$ filter coefficients. Thus, the amplitude of the received peak decreases by the sum of the square coefficients in the shifted part of the transmitted signal. Therefore with the new modulation scheme, the received signal peak reduces in proportion to the energy of the shifted part of the transmitted signal.

Fig. 2 shows the peak power of the received signal peak for the shifted signals normalized to the received peak with no shift. The shift percentage corresponds to the percentage of the total length of the transmitted signal. A set of 243 measured CIRs are used for the simulation. Experimental setup and the measurement procedure are explained in Section IV. The loss of the received peak power for transmitted signals corresponding to individual CIRs is represented by the dots and the dashed line is the mean of power loss. To calculate the maximum number of simultaneous users that a system can support, we must take the decision in accordance to the threshold (say $3 \mathrm{~dB}$ ) which can vary for different applications. For a $3 \mathrm{~dB}$ threshold, our system can support a shift percentage 


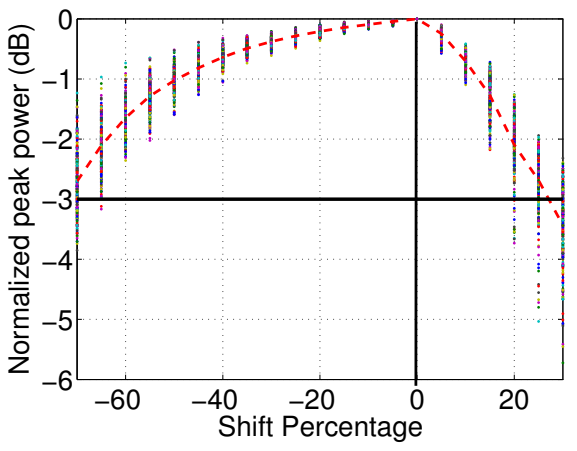

Fig. 2. Received signal peak power with left and right shifts normalized to the peak with no shift

of $0.70 \mathrm{~L}$ taps for left shift and $0.25 \mathrm{~L}$ taps for right shift (see Fig. 2). Thus, the number of users with the proposed scheme can be written as:

$$
N_{u}^{\text {mod.TR }}=\left\lfloor\frac{0.95 \times L}{\delta}\right\rfloor=\left\lfloor\frac{0.95 \times T_{\text {sig }}}{\Delta T R}\right\rfloor
$$

where $\lfloor$.$\rfloor denotes the floor operator, L$ is the total number of taps in the transmitted signal, $\delta$ is the shift percentage between two simultaneous users, $T_{\text {sig }}$ is the channel length in $s$ and $\Delta T R$ is shift separation between two users in $s$. For the same threshold, the previously proposed scheme in [8] can only support a shift of $0.75 \mathrm{~L}$ which is contrary to their claim of $100 \%$ shift (as power loss with circular shift operation was not considered by the authors). The power loss for left shift is lesser than the power loss for the right shift as the energy contained in the shifted parts of the right shift is greater than the energy contained in the shifted parts of the left shift. Although a combination of right and left shifts can be used for the communication, for the sake of simplicity we have only used left shift. In the rest of the paper unless otherwise mentioned, a shift is meant to be a left shift.

Power spectral density (PSD) of the transmitted signal of a TR communication system takes into account the effects of the antennas and the propagation channel including the path loss. In contrast to the pulse signal, the spectrum of a TR signal has a descending shape with increasing the frequency because the higher frequency components experience a greater path loss as compared to the lower frequency components in the spectrum. Fig. 3 shows the PSD plots of the transmitted signal with simple TR and modified TR schemes where a left shift of $0.20 \mathrm{~N}$ taps is carried out for both modified TR scheme. The plots of both schemes have a descending shape. Maximum spectral power is experienced at the same frequency. Therefore, both the signals must be attenuated with the same factor in order to respect the UWB spectral mask proposed by FCC. Frequency selectivity of the transmitted signals is similar for the two schemes. In short, the both schemes have resembling spectral properties.

\section{EXPERIMENTAL Setup AND Simulation Results}

Experiments are performed in a typical indoor environment. The environment is an office space of $14 \mathrm{~m} \times 8 \mathrm{~m}$ in the

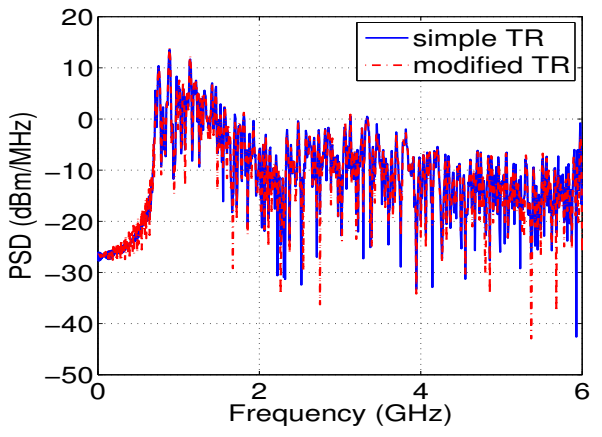

Fig. 3. PSD of transmitted signal with simple TR and modified TR schemes

IETR $^{1}$ laboratory. The frequency response of the channel in the frequency range of $0.7-6 \mathrm{GHz}$ is measured using vector network analyser (VNA) with a frequency resolution of 3.3 $\mathrm{MHz}$ and two wide band conical mono-pole antennas (CMA) in non line of sight (NLOS) configuration. The height of the transmitter antenna and the receiver antenna is $1.5 \mathrm{~m}$ from the floor. The receiver antenna is moved over a rectangular surface $(65 \mathrm{~cm} \times 40 \mathrm{~cm})$ with a precise positioner system. The frequency responses between the transmitting antenna and receiving virtual array are measured. The time domain CIRs are computed using the inverse fast Fourier transformation (IFFT) of the measured frequency responses.

\section{A. BER Performance}

In the proposed transmission scheme, one user is separated with the other by a shift of a fixed number of taps. This separation is named as $\delta$, which is a percentage of total number of taps in the transmitted signal. Signal for User 1 is transmitted without any shift. As discussed in Section III-A, that interference between users is greatly reduced with the proposed modulation scheme. To study the impact of the reduced interference, we evaluate the BER performance with the proposed scheme using left shift for 5, 10 and 15 simultaneous user for $\delta=0.05 L$.

From the measured CIRs, we generate almost $35 \times(35-$ $\left.N_{u}-1\right)$ combinations for simulating different number of simultaneous users $\left(N_{u}\right)$. For every combination of simultaneous users, 10000 symbols are transmitted which makes it sufficient for statistical analysis. The measured CIR is truncated for $90 \%$ energy contained in the CIR. Thus, the transmitted symbol has a length of $55 \mathrm{~ns}$ and a per user bit rate of $18 \mathrm{Mbps}$. Perfect synchronization and no ISI effects are assumed. Signal to noise ratio (SNR) is varied by varying the noise variance, as:

$$
S N R=P_{j} / \sigma_{\text {noise }}^{2}
$$

where $P_{j}$ is the power of the received signal at its peak and $\sigma_{\text {noise }}^{2}$ is the noise variance. Bipolar pulse amplitude modulation (BPAM) is used for these simulations. The received signal $y_{j}(t)$ is sampled at its peak and is detected based on

\footnotetext{
${ }^{1}$ Institute of Electronics and Telecommunications of Rennes
} 


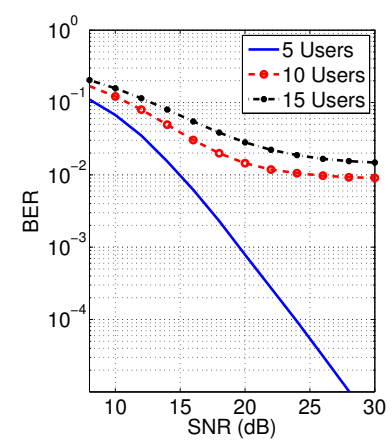

(a)

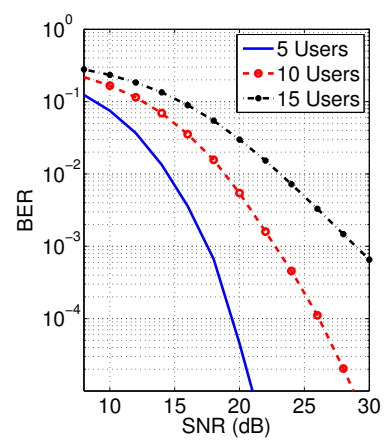

(c)

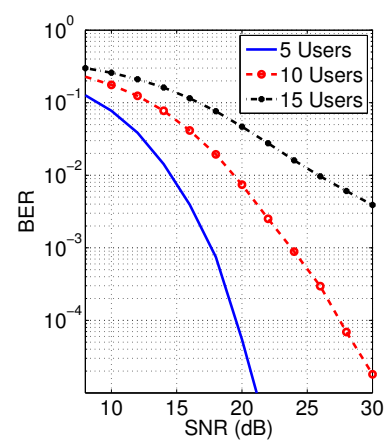

(b)

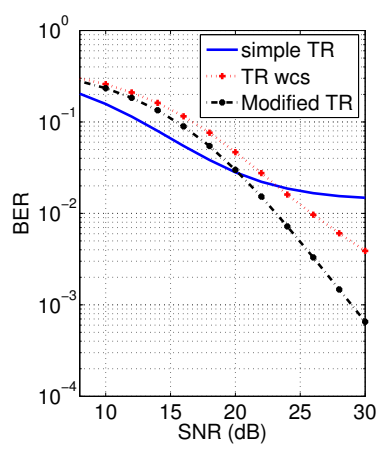

(d)
Fig. 4. BER performance with 5, 10, and 20 simultaneous users with a) simple TR b) TR with circular shift c) modified TR scheme d) 15 simultaneous users with all three schemes for $\delta=0.05 L$ taps

ideal threshold detection, given as:

$$
\text { Detected bit }= \begin{cases}1 & \text { if } y_{j}\left(t_{\text {peak }}\right) \geq 0 \\ 0 & \text { if } y_{j}\left(t_{\text {peak }}\right)<0\end{cases}
$$

Fig. 4a-c shows the BER performance of the simple TR, TR with circular shift operation and the modified TR scheme for 5, 10 and 15 simultaneous users. The modified TR scheme outperforms the other two schemes specially for higher number of simultaneous users $(10,15)$. For instance for 10 simultaneous users, the modified TR scheme results in a $1.4 d B$ better performance than the TR with circular shift for a BER of $10^{-4}$. The simple TR scheme has already reached a plateau. To perform an analysis in the presence of extreme multi user interference, BER performance is studied for 15 simultaneous users. Fig. 4d compares the performance of the three schemes for 15 simultaneous users. The modified TR scheme gives significantly better performance than the other two schemes. The improvement is in the order of $4.5 \mathrm{~dB}$ or more.

If a system has large number of users, the users experiencing higher shift percentages will give poorer performance than the users experiencing lower shift percentages. To have a consistent system, we propose to rotate the shift percentages for different users so that no user is subjected to permanent high shift percentage.

\section{Conclusion}

In this paper, a modified transmission scheme for a multi user time reversal system is proposed. With the help of mathematical derivations, it is shown that the interference in the modified TR scheme is reduced compared to simple TR scheme. Limitations of the proposed scheme are studied and an expression for maximum number of simultaneous users is proposed. It is shown that the modified TR scheme outperforms simple TR and TR with circular shift scheme specially at higher number of simultaneous users. For instance for 15 simultaneous users, the modified TR scheme improves the performance in the order of $4.5 \mathrm{~dB}$ or more for a constant BER.

\section{REFERENCES}

[1] R. Hoctor and H. Tomlinson, "Delay-Hopped Transmitted-Reference RF Communications," Proc. IEEE 2nd Ultra Wideband Systems and Technologies (UWBST02), Baltimore, MD, pp. 265-269, May 2002.

[2] R. J. Fontana, E. Richley, and J. Barney, "Commercialization of an Ultra Wideband Precision Asset Location System," Proc. IEEE Conference UWB systems and Technologies, Reston, VA, 2003.

[3] J. D. Choi and W. E. Stark, "Performance of Ultra-Wideband Communications with Suboptimal Receivers in Multipath Channels," IEEE Journal on Selected Areas in Communications, vol. 20, pp. 1754-1766, Dec. 2002.

[4] M. Z. Win and R. A. Scholtz, "Impulse radio: How it works", IEEE Commun. Lett., vol. 2, no. 2, pp. 36-38, Feb. 1998.

[5] M. Z. Win and R. A. Scholtz, "Characterization of ultra-wide bandwidth wireless indoor channels: A communication-theoretic view ", IEEE J. Select. Areas Commun. (JSAC), vol. 20, no. 9, pp. 1613-1627.

[6] M. Fink, "Time-reversed acoustic", Scientific Amer., pp. 67-73, Nov.1999.

[7] A. Derode, A. Tourin, J. D. Rosny, M. Tanter, S. Yon, and M. Fink, "Taking advantage of multiple scattering to communicate with timereversal antennas", Phys. Rev. Lett., vol. 90, no. 1, pp. 10143011014301-4, 2003.

[8] H. T. Nguyen, I. Z. Kovcs,P. C.F. Eggers, "A Time Reversal Transmission Approach for Multiuser UWB Communications", IEEE Transactions On Antennas and Propagation, Vol. 54, NO. 11, November 2006.

[9] P. Kyritsi, G. Papanicolaou, P. Eggers, and A. Oprea, "MISO Time Reversal and Delay-Spread Compression for FWA Channels at $5 \mathrm{GHz}$ ", IEEE Antennas Wireless Propag. Lett., vol. 3, no. 6, pp. 96-99, 2004.

[10] P. Kyritsi, G. Papanicolaou, P. Eggers, and A. Oprea, "One-bit Time Reversal for WLAN Applications", Personal, Indoor and Mobile Radio Communications, 2005. PIMRC 2005.

[11] A. E. Akogun, R. C. Qiu, and N. Guo, "Demonstrating time reversal in ultra-wideband communications using time domain measurements," in 51st Int. Instrument. Symp., Knoxville, TN, May 8-12, 2005.

[12] H. T. Nguyen, J. B. Andersen, and G. F. Pedersen, "The Potential Use of Time Reversal Technique in Multiple Elements Antenna Systems", IEEE Commun. Lett., vol. 9, no. 1, pp. 40-42, Jan. 2005.

[13] T. Strohmer, M. Emami, J. Hansen, G. Papanicolaou, and A. J. Paulraj, "Application of time-reversal with MMSE equalizer to UWB communications", in Proc. IEEE Global Telecommunications Conf. (GlobeCom), Dec. 2004, vol. 5, pp. 3123-3127.

[14] R. C. Qiu, C. Zhou, N. Guo, and J. Q. Zhang, "Time Reversal with MISO for Ultra-Wideband Communications: Experimental Results", IEEE Antenna and Wireless Propagation Letters, 2006.

[15] A. Khaleghi, G. El Zein, I. H. Naqvi, Demonstration of Time-Reversal in Indoor Ultra-Wideband Communication: Time Domain Measurement, ISWCS 2007, Trondheim, Norway

[16] A. Khaleghi, G. El Zein, "Signal Frequency And Bandwidth Effects On The Performance Of UWB Time-Reversal Technique", Loughborough Antennas and Propagation Conference 2007, Loughborough, UK.

[17] I. H. Naqvi, A. Khaleghi, G. El Zein "Performance Enhancement of a Multiuser Time Reversal UWB Communication System", International Symposium on Wireless Communication Systems 2007, Trondheim, Norway. 